\title{
Disseminated Tuberculosis Causing Pancytopaenia in an Indian Boy
}

\author{
Roy $\mathrm{A}^{1}$, Chaudhuri J², Kumar $\mathrm{K}^{3}$, Mukhopadhyay $\mathrm{D}^{4}$ \\ ${ }^{1}$ Dr. Amrita Roy, MBBS, MD Resident (Paediatrics). \\ ${ }^{2}$ Dr. Jasodhara Chaudhuri, MBBS, MD Resident \\ (Paediatrics), ${ }^{3} \mathrm{Dr}$. Kishore Kumar, MBBS, MD, DM, \\ Institute of Hematology and Transfusion Medicine. \\ ${ }^{4}$ Dr. Debadutta Mukhopadhyay, MBBS, MD, Assistant \\ Professor of Paediatrics. All from Medical College, \\ Kolkata.
}

\section{Introduction}

Tuberculosis is still a major public health problem in developing countries. Patients with disseminated tuberculosis have varied presentations including pyrexia of unknown origin, hepatosplenomegaly, lymphadenopathy, and meningitis ${ }^{1}$. Though numerous haematological abnormalities have been associated with tuberculosis, bleeding manifestations and ascites as presenting symptoms along with bone marrow infiltration by tubercular granuloma causing pancytopaenia is uncommon.

\section{The Case}

Eleven year old boy presented with six weeks' history of low grade fever, weight loss, anorexia, petechial spots and progressive abdominal distention. There was no history of gastro-intestinal tract bleeding or cough. There was no past history of malaria or blood transfusion. He was not on any chronic medication. However, his grandfather was treated for sputum negative pulmonary tuberculosis six months back.

On examination, he was cachectic, anaemic with huge hepatomegaly (liver smooth, palpable $15 \mathrm{~cm}$ below right subcostal margin), splenomegaly (palpable $1 \mathrm{~cm}$ ), ascites and multiple petechial spots over his trunk, diminished vesicular breath sounds over right hemithorax with dull percussion note. There was no icterus or lymphadenopathy or any stigmata of chronic liver disease.

His complete blood counts on admission showed haemoglobin of $6.1 \mathrm{gm} / \mathrm{dl}$, Total Leucocyte Count of

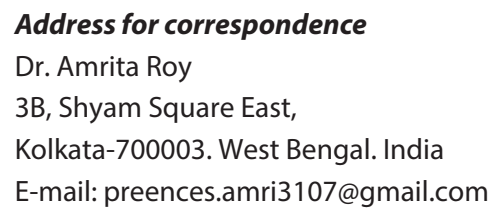

\begin{abstract}
Tuberculosis still remains a major public health problem, particularly in developing countries. We report 11 year old Indian boy who presented with low grade fever, weight loss, hepatomegaly, ascites and petechial spots for past 6 weeks associated with pancytopaenia. Subsequently, bone marrow biopsy from the iliac crest revealed an epithelioid cell granuloma with hypercellularity. This is a rare presentation where disseminated tuberculosis presented as pancytopaenia.
\end{abstract}

$1800 / \mathrm{cu}$. mm, lymphocytes $65 \%$, neutrophils $32 \%$, eosinophils $2 \%$, monocytes $1 \%$, platelet count of $30,000 /$ cu. $\mathrm{mm}$, reticulocyte count $0.1 \%$. RBC indices showed MCV 92 fl, MCH 25.8 pg, MCHC 32.4 gm/dl, RDW 15.4. Peripheral blood smear showed anisocytosis, poikilocytosis, round macrocytes, microcytes, tear cells, pencil cells; leucocytes showed shift to left till myelocyte stage. ESR was $140 \mathrm{~mm} / 1^{\text {st }}$ hour. His liver function tests revealed Albumin $2.8 \mathrm{~m} / \mathrm{dl}$, Globulin $3.2 \mathrm{gm} / \mathrm{dl}$; normal SGOT, SGPT; total bilirubin $1.2 \mathrm{mg} / \mathrm{dl}$ and alkaline phosphatase (921 IU/l; normal <800 IU/L). Coagulation profile showed INR 1.11and APTT 15.2 secs. Serum urea, creatinine and electrolytes were within normal limits.

Serum for HBsAg, anti HCV, anti HAV was negative. The PPD test was strongly positive $(18 \mathrm{~mm}$ x $18 \mathrm{~mm}$ ). His chest radiograph revealed right sided pleural effusion. HRCT Chest also corroborated with the findings along with right hilar and mediastinal lymphadenopathy. Sputum smear for acid fast bacilli was negative. Ultrasonography of the abdomen showed enlarged liver with homogeneous echotexture, multiple periportal and mesenteric lymph nodes with $450 \mathrm{ml}$ of free fluid but no evidence of portal hypertension.

The preliminary diagnosis was of a haematologically malignant condition like lymphoma infiltrating the bone marrow complicated by an opportunistic infection with Mycobacterium tuberculosis. The differentials included chronic liver disease with hypersplenism due to portal

\section{How to cite this article?}

Roy A, Chaudhuri J, Kumar K, Mukhopadhyay D. Disseminated Tuberculosis Causing Pancytopaenia in an Indian Boy. J Nepal Paediatr Soc 2013;33(1):52-54. 
hypertension, Systemic Lupus Erythrematosus, Wilsons disease.

Ascitic fluid was of exudative (SAAG<1.1), ADA $96 \mathrm{IU} / \mathrm{L}$, no malignant cells. The pleural fluid had ADA $37 \mathrm{IU} / \mathrm{L}$ with negative malignant cytology. Blood culture and Coomb's test was negative. HIV I \& HIV II Antibody ELISA was negative. His erythrocyte folate level was below normal with normal serum Vitamin B 12. Upper GI endoscopy did not reveal any esophageal or gastric varices. Haemophagocytic lymphohistiocytosis was excluded by serum ferritin $424 \mathrm{ug} / \mathrm{l}$, TAG $125 \mathrm{mg} /$ dl. Serum ANA and anti-dsDNA was negative ruling out autoimmune pathology like Systemic Lupus Erythrematosus. Screening for Wilson's disease was negative.

Bone marrow biopsy from iliac crest showed hypercellularity and multiple ill defined epitheloid cell granulomas, areas of necrosis, diffuse presence of plasma cells and macrophages as shown in Figure 1. Reticulin network was diffusely increased. Bone marrow iron store was normal.

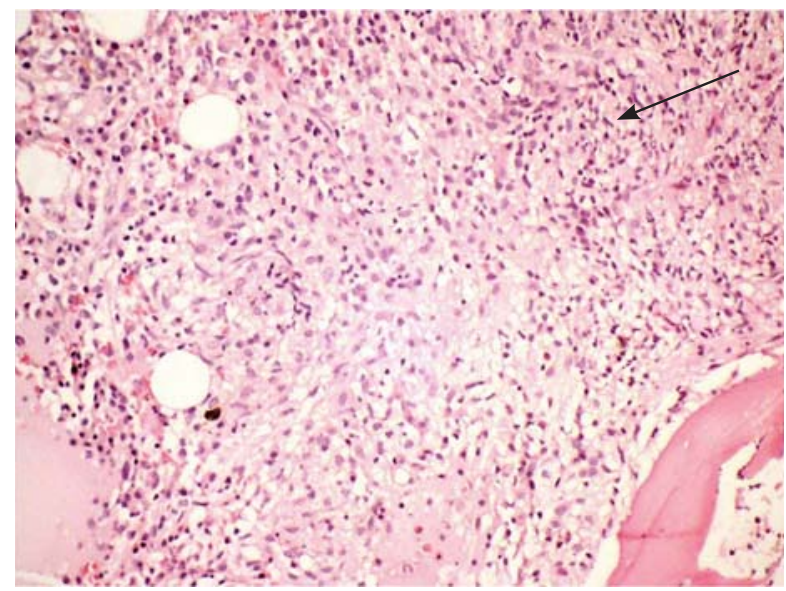

Fig 1: Showing 40x magnification Haematoxylin Eosin stain of Trephine biopsy section showing collections of epithelioid cells forming ill-defined granulomas (shown by arrows)

The patient was conservatively manged with Packed Red Cells and platelets transfusion and antitubercular drugs (ATD). The patient improved within two weeks of anti-tubercular drugs with disappearance of fever, cessation of bleeding, regression of ascites and his complete blood count after four weeks of ATD treatment showed haemoglobin of $11.2 \mathrm{~g} / \mathrm{dl}$, TLC 7200/ cu $\mathrm{mm}$, neutrophils $42 \%$, lymphocytes $56 \%$, eosinophils $2 \%$, platelet count $1,80,000 / \mathrm{cu}$ mm. Repeat bone marrow biopsy did not reveal any tubercular granuloma at the end of treatment. Thus the counts showed a significant improvement with the institution of ATD showing that the pancytopenia was due to tuberculosis disease process itself with no other associated pathology.
The patient is currently under follow up and after 1 year, he is doing well with no evidence of any haematological abnormality.

\section{Discussion}

The term "disseminated" refers to active haematogenous spread of Mycobacterium tuberculosis in two or more organs/systems in the body leading to a generalized systemic illness. It is characterized by little tissue reaction with multiple acid-fast bacilli reflecting failure of cell-mediated immunity. When a caseous focus empties into a vein, it overwhelms host defences and tuberculous granulomas are established in almost all organs $^{2}$.

The symptoms and signs of miliary TB are nonspecific $^{2}$ including fever, weight loss, dyspnoea, cough. Miliary TB can imitate chronic infections like histoplasmosis and cryptococcosis ${ }^{2}$. The PPD skin test (with 5 tuberculin units) is only positive in $75 \%{ }^{3}$. Radioisotope scans and CT of the liver and spleen are of little help if the granulomas remain small ${ }^{3}$. Chest $X$ ray findings may show miliary consolidation, pleural effusion or mediastinal gland enlargement or calcification. There is often a raised alkaline phosphatase level with normal transaminase levels as obtained in our patient. Miliary tuberculosis may be diagnosed by needle biopsies of the lung and/or liver, mediastinoscopy, laparoscopy or laparotomy ${ }^{2}$.

Anaemia is the most common haematolorical disorder caused by TB but other abnormalities occur $^{4}$ such as leucopenia, leukaemoid reaction, myelofibrotic changes, the haemophagocytic syndrome, polycythaemia, thrombocytosis, monocytosis, basophilia, disseminated intravascular coagulopathy, sideroblastic and megaloblastic anaemia are reported. White cell counts are usually slightly reduced or within normal limits with a 'left shift'. Folate levels are frequently reduced, reflecting the poor physical condition and dietary deficit. Bleeding manifestations and/or pancytopenia as a presenting feature of disseminated tuberculosis is rare in children and adults. Pancytopenia is the rarest of the haematological findings ${ }^{5}$ and its degree is influenced more by duration of the infection than its severity.

Pancytopenia was seen only in $8 \%$ in a series of 38 patients with miliary tuberculosis by Mert et al ${ }^{6}$ but there was no episode of significant bleeding. Granulomas were found in $56 \%$ patients' bone marrow tissue specimen, and polymerase chain reaction (PCR) was positive in $47 \%$ specimens with granuloma ${ }^{6}$. Though it was thought that subjects with caseating granuloma carry very poor prognosis $^{1}$, in our case ATD has altered the course of the disease. Cooper ${ }^{7}$ has described a patient where 
pancytopenia resolved with the removal of an enlarged tuberculous spleen. Otherwise pancytopenia attributable to tuberculous hypersplenism has rarely been seen. Most cases of pancytopenia with tuberculosis are due to an underlying haematological malignancy like myelodysplastic syndrome and was preterminal ${ }^{4}$.

Sabin et al. ${ }^{8}$ showed that animals with disseminated tuberculosis involving the marrow developed pancytopenia. This hypothesis was supported by cases where there was recovery of peripheral blood counts with ATD ruling out haematological disease.

Tuberculosis can cause pancytopaenia due to the toxiceffect oftuberculin on the bonemarroworgranulomas causing fibrosis ${ }^{2}$. Tubercular granuloma can replace marrow cells or depress marrow and cause maturation arrest through certain interferons and cytotoxins. Pancytopaenia may be due to hypersplenism, histiocytic hyperplasia and phagocytosis ${ }^{2}$. Thrombocytopenia can occur due to the accumulation of mycobacterial antigen on platelet surfaces.

In miliary TB and pancytopenia, bone marrow aspiration usually reveals normal or raised cellularity and only occasionally is there a true aplasia.From reported literature, when TB occurs with a pancytopenia, the mortality is very high in spite of ATD².

In any patient with a pyrexia of unknown origin and a raised serum alkaline phosphatase level, the diagnosis of tuberculosis must be considered. If the patient's condition does not allow a needle biopsy of the liver and/or lung or a laparoscopy, a therapeutic trial with ATD must be instituted ${ }^{2}$.

Anti-tuberculous drugs may alter the haematological picture due to idiosyncratic side effects. Rifampicin may cause an immune haemolytic anaemia and/or thrombocytopenia. Many drugs such as pyrazinamide produce a sideroblastic anaemia, whilst idiosyncratic episodes of agranulocytosis or aplastic anaemia have occurred with streptomycin.

Treatment of an anaemia or pancytopenia with steroids in the presence of TB may lead to the dissemination of the bacilli and the development of miliary disease. Steroids should therefore be used with care until the possibility of TB has been excluded.

Patients with pancytopenia with tuberculosis have a high mortality, but there are a few that have recovered. Mangion ${ }^{10}$ described the only documented case where tuberculous pancytopenia recovered fully and the patient was followed for 9 months. Our patient also had pancytopaenia due to tuberculosis and has recovered completely with ATD.

\section{Conclusion}

Tuberculosis, a major cause of morbidity and mortality can have varied presentations. This 11 year old Indian boy who presented with low grade fever, hepatomegaly, ascites and petechial spots, pancytopaenia for past six weeks with pancytopaenia had ill-defined tubercular granulomas in bone marrow trephine biopsy which is a very rare presentation of disseminated tuberculosis. The patient responded with anti-tubercular drugs within two weeks and contrary to reported cases where caseating granulomas had a very poor prognosis, our case recovered completely.

Disseminated tuberculosis associated with pancytopaenia is a very rare presentation with a handful of reported cases. But contrary to most of the reported cases, our patient recovered completely with antitubercular drugs. So, a trial of ATD should always be considered.

\section{References}

1. Yadav TP, Mishra S, Sachdeva KJS et al. Pancytopenia in disseminated tuberculosis. Indian Pediatr. 1996;33:597-9.

2. Mario C. R., Richard J. O'Brien. Mycobacterial diseases-tuberculosis. Harrison's Principles of Internal Medicine. 18th ed. London: McGraw-Hill Medical. USA. 2012:1340-1366.

3. Knobe1 B, Sommer I, Saide1 L, Robson M. Pancytopenia: a rare complication of miliary tuberculosis. Isr J Med Sci. 1983;19:555-558.

4. Glasser RM, Walker RI, Herion JC, Hill C. The significance of hematologic abnormalities in patients with tuberculosis. Arch Intern Med. 1970;125:691-95.

5. Katzen H, Spagnolo SV. Bone marrow necrosis from miliary tuberculosis. JAMA. 1980;244:24382439.

6. Mert A, Bilir M, Tabak F et al. Miliary tuberculosis: Clinical manifestations, diagnosis and outcome in 38 adults. Respirology. 2001;6:214-24.

7. Cooper, W., Pancytopenia associated with disseminated tuberculosis. Ann Intern Med. 1959,50:1497-1501.

8. Sabin FR, Doan CA, Forkner CE. Studies on tuberculosis. J Exp Med. 1930, supp 13:1-152.

9. Kashyap S, Puri DS, Bansal SKetal. Mycobacterium tuberculosis infection presenting as pancytopenia with hypocellular bone marrow. J Assoc Phys India. 1991;39:497-8.

10. Mangion, P.B. Disseminated tuberculosis complicated by pancytopenia. Proc $R$ Soc Med. 1971;64:1000. 